Physical Sciences | David J. E. Marsh

\title{
Searching for axions
}

Revealing the dark matter particle

Dark matter is one of the central mysteries of modern cosmology. Even after many years of investigation into the true natur of this enigmatic component of our Universe, every search for its cause has so far come up short. Dr David J. E. Marsh believes that the solution lies with a fundamental particle which was first theorised over 40 years ago, but due to the limitations of technology in the past, has not yet been detected. He has proposed a new type of experiment which will soon join the worldwide hunt for this

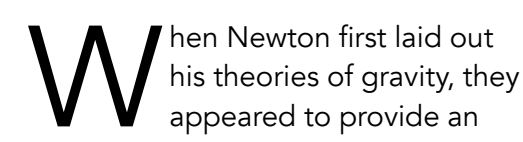
elegant description of how the stars and planets moved through the solar system. As astronomers came to view the Universe on larger scales, howev this comfortable picture changed entirely. From the rotation speeds of galaxies to the rate of the Universe's realised that many of the Universe's characteristics could only make sense if it contained vast amounts of some seemingly invisible material.

Dubbed 'dark matter,' this mysterious substance is now believed to comprise around $85 \%$ of all matter in the Universe; yet despite its prominence, its true nature remains completely unknown. "Thanks to astrophysica observations, it is known that dark matter permeates the Universe, and is composed of a new type of particle, University of Göttingen's Dr Marsh explains. The question of what this particle could be has long captivated quarks make up today's "standard model",

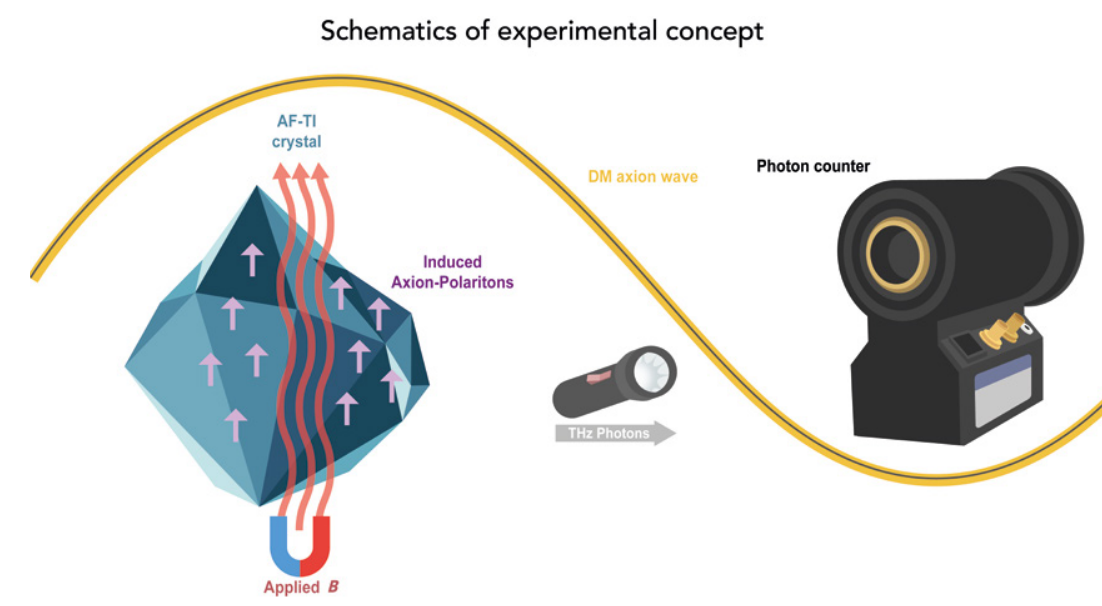

Dr Marsh's proposed Topological Resonant Axion Detection
tell-tale signatures of axions as they pass through the Earth. researchers know that dark matter does not interact in the usual ways using the three fundamental forces of wectromagnetisn and the strong and

So far, most theories have had virtually no success in explaining model favoured by the community was the weakly interacting massive particle, or "WIMP'", continues Dr Marsh "However, past series of experiments have failed to find WIMPs, and the fortunes of its paren theory, supersymmetry, have also fallen on hard times." Although the search for WIMPs still persists, another theory has recently gained traction which researchers like Dr Marsh believe could provide an equally promising explanation.

\section{EXPANDING THE SEARCH}

FOR AXIONS

In the late 1970s, new calculations of the interactions which take place with the protons and neutrons of atomic new fundamental particlo an enty the 'axion.' Initially, the idea wasn't conceived to explain the nature of dark matter. However, researchers quickly realised that if axions had low, specific masses as their calculations suggested they could well make up a significant proportion of the Universe's mass; all while going completely undetected by particle detectors on Earth.

Our knowledge of axions remains confined to theory for now, but Dr Marsh and his colleagues - along with many other research groups - are now aiming to change this. In recent years, search for axions have beenn cons to search for axions have been conceived, close to realisation," he describes.
Where typical particle physics Werperticles new discoveries, axion detectors are now becoming increasingly sophisticated through improvements in measurement technologies. For Dr Marsh and his team, these advances have paved the way for the 'Topological Resonant Axion Detection' (TOORAD) experiment - a proposed experiment which aims to pick up the tell-tale signatures of axions as they pass through the Earth.

FINDING THE RIGHT FREQUENCY Dr Marsh's approach has joined a growing list of studies that are now on the hunt for axions. Together, different around theoreticat calcultions of the nature of axions. However, since these theories have so far been unable to pin down the exact properties of the particles, it is currently far from clear which of them will work. Essentially, if a discovery is made, it will be through trial and error of different technologies.

Searching for the axion is like tuning a radio," Dr Marsh illustrates. "It

needs a specific frequency, but we

don't know what that frequency is,

although astrophysics tells us the minimum and maximum range. Tha radio we need to buld You we of find the station, but you don't know if

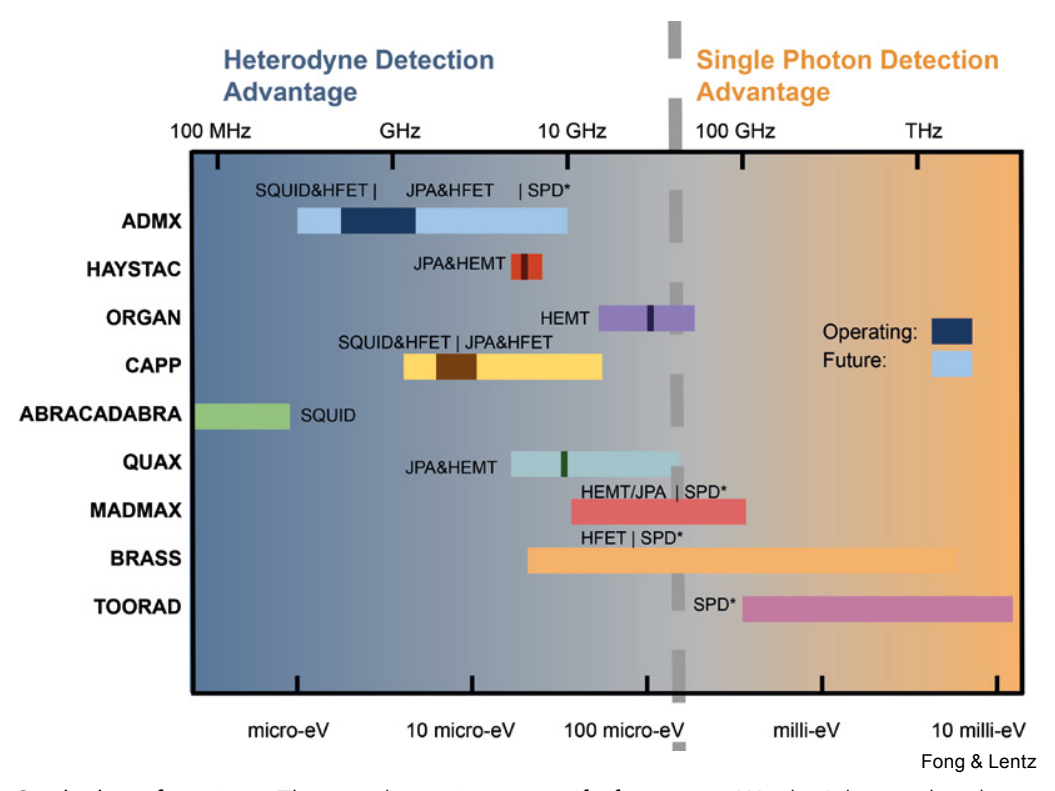

On the hunt for axions: The search requires a specific frequency. We don't know what that

Our knowledge of axions remains confined to theory for now, but Dr Marsh and his colleagues aim to change this.

you need an FM or longwave radio, or even an $x$-ray machine."

A WIDE RANGE OF EXPERIMENTS Among the many experiments their own specific frequency ranges is MADMAX - an arrangement of several adjustable, parallel disks, made from insulating materials whose positive and negative charges to an electric field. Current theories suggest that if an axion of a specific his arty range were to pass thagnetic waves it produces would bounce back and forth between the disks. This would greatly amplify the virtually imperceptible signal, alerting researchers to the axion's presence.

Another experiment operating in a different frequency range is distinguishing wo whes in the spins of

Axions verses WIMPs
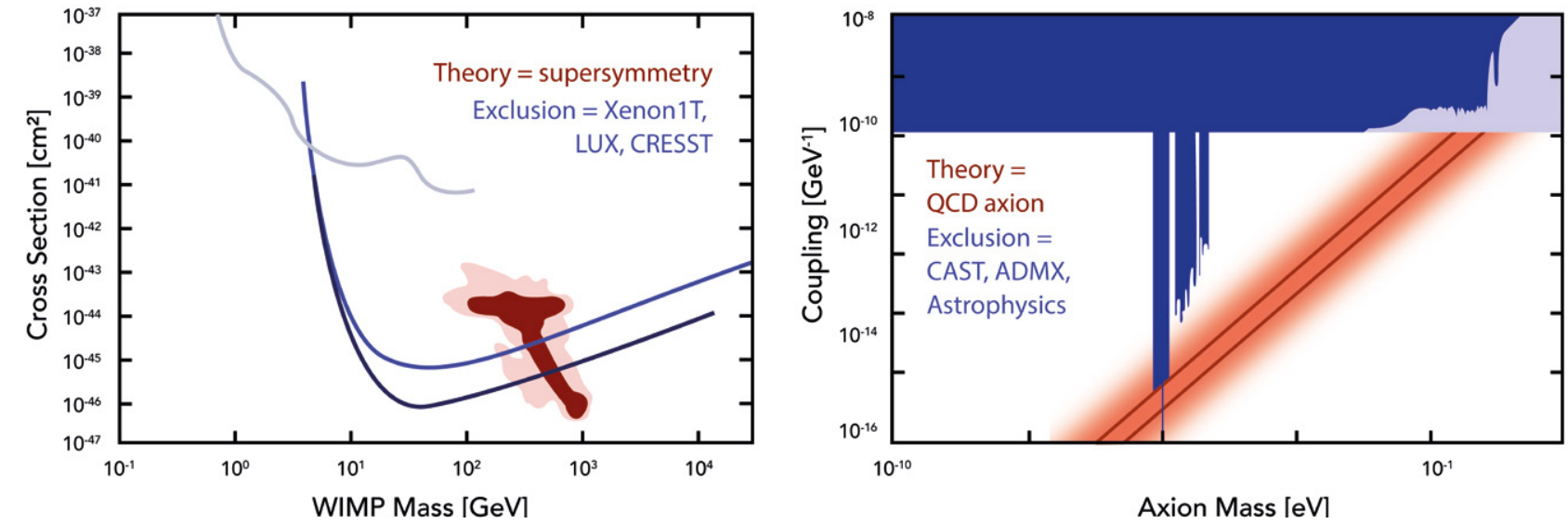

.
Axion Mass [eV] 


\section{Detection: Topological insulators}

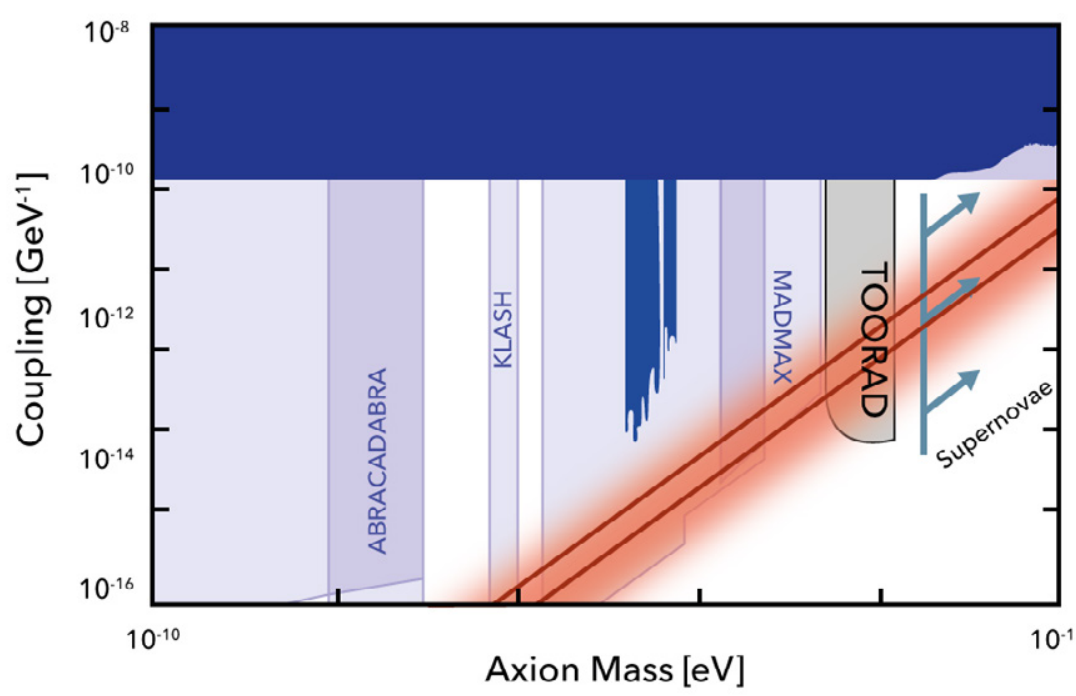

Projections for the future of axion searches. Image adapted from https://github.com/cajohare/
Axionlimits

tomic nuclei that have been aligned by strong magnetic fields, which would occur if an axion passed through them. Afterwards, the spins would re-align themselves with the magnetic field giving off electromagnetic waves an axion had been detected.

Comparably, the QUAX experiment aims to detect characteristic disturbances in ferromagnets. Thes metals have atomic spins which are astrophysics and particle physics.

EXPLORING THE UPPER RANGE TOORAD targets the higher end of the frequency range that current axion theories allow for. To detect these particles, Dr Marsh and his colleagues will need to develop a material which doesn't obey the usually strict rules describing how electromagnetic waves interaction

\section{The technologies draw on materials} science, high energy physics, precision measurement, and theoretical astrophysics and particle physics.

would again be disturbed if an axion of the right frequency range passed through, altering the material's magnetisation in turn. Along with many other experiments, "these effor are being constructed now all over the world, with activity concentrate in Germany, South Korea, and the USA," Dr Marsh summarises. "The tech hologies draw on materials

first demonstrated experimentally in 2007. Named 'topological insulators,' they possess exotic arrangements of electrons which permit changes to the laws of electromagnetism inside them. In TOORAD, topological insulators are combined with 'antiferromagnetic' materials - containing atoms whose spins are aligned like ferromagnets, but
which always point in exactly opposite directions to their neighbours. With hese combined properties, Dr Marsh's axions with terahert frequencies would, thanks to the laws of equectromagnetism allowed in topological insulators, set the antiferromagnetic spins oscillating in a "spin wave". The spin wave itself then leads to emission of electromagnetic waves from the material with a tiny, but detectable, power output

TOORAD remains in the design stages for now, and isolating a material with just the right structure and composition will be no easy task for Dr Marsh's

team. Yet through a rigorous research campaign, the researchers believe the will be able to develop just the right antiferromagnetic topological insulator haven't found the perfect mas. have but we the working with materials cientists to find it," Dr Marsh explains. "This means growing entirely new compounds, and testing them at $\mathrm{THz}$ frequencies, ultracold temperatures, and in high magnetic fields."

\section{WAITING FOR}

CONCLUSIVE RESULTS

Dr Marsh is now hopeful that while

previous dark matter theories have

suffered from a lack of experimenta

evidence, the search for axions has only

just begun, and promises to massively

ramp up in the coming years.

"So far, very few axion experiments have been able to search in the righ will all change. If the axion exists, ot of these new ideas will find it But, due to the different frequency ranges of each technology, we aren't in direct competition. Nature will determine who chose the right design," he concludes.

Through TOORAD and its many contemporaries which search for axions at different frequency ranges, astronomers could soon be able to finally confirm the true nature of the majority of matter our Universe. If just one of these experiments yields a positive result, the answers it provides could provide the first satisfying answers to long-standing cosmological mysteries ranging from how will expand into the distant funte.

\section{Behind the Research}

\section{Dr David J. E. Marsh}

W: https://djemarsh.wixsite.com/physics W: https://djemarsh.wixsite.com/cosmologylive

Research Objectives

David Marsh's research explores the intersection of Cosmology and High Energy Physics. Much of his

\section{Detail}

David Marsh, Institute für Astrophysik

Friedrich-Hund PI. 1, Göttingen 37077, Germany

Darsh was born in Liverpool and attended Edinburgh University. He did his PhD in Oxford on theoretical particle physics and cosmology, thesis "The String Axiverse and Cosmology". David is currently a Sofja Kovalevskaja fellow in Germany. Outside of physics he has been skateboarding for more than 20 years and loves literature and reggae music.

Funding

The Alexander von Humdbolt Foundation The German Federal Ministry of Education and Research

Collaborators

All my co-authors, especially Mazhar Ali. All my colleagues, those currently pushing TOORAD collaboration, Jan Schuette-Engel, and the QUAX TOORAD interest group.

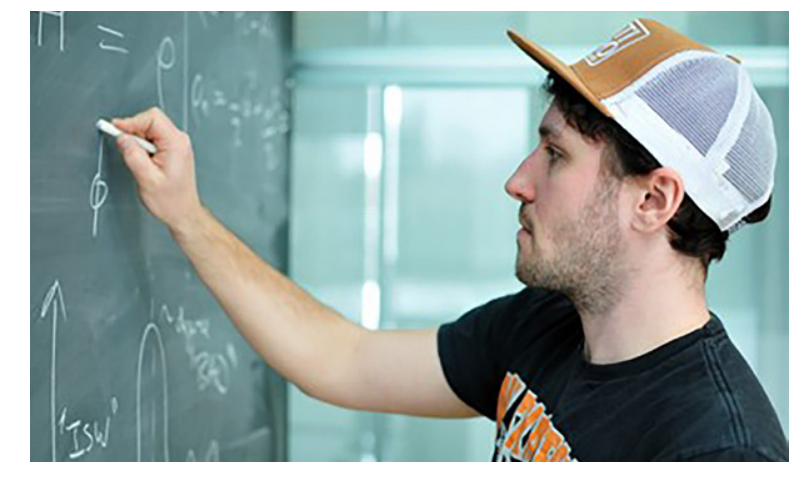

INSTITUT FÜR

ASTROPHYSIK GöttINGEN

\section{References}

- Marsh, D.J.E. (2016). Axion cosmology. Physics Reports, 643, 1-79. arXiv:1510.07633

- Kimball, D.F., Afach, S., Aybas, D., Blanchard, J.W., Budker, D., Centers, G., Engler, M., Figueroa, N.L.,

Garcon, A., Graham, P.W., Luo, H. (2017). Overview of the cosmic axion spin precession experiment (CASPEr). arXiv preprint, anXiv.1711.08999

- Brun, P., Caldwell, A., Chevalier, L., Dvali, G., Freire, P., Garutti, E., Heyminck, S., Jochum, J., Knirck, S., Kramer, probe $\mathrm{OCD}$ axion dark matter in the mass range above 40ueV. The European Physical Journal C. 79(3), 186. arXiv:1901.07401

- Crescini, N., Alesini, D., Braggio, C., Carugno, G. Di Gioacchino, D. Gallo, C.S. Gambardella, U., Gatti, C lannone, G., Lamanna, G., Ligi, C. (2018). Operation of a ferromagnetic axion haloscope at $\mathrm{m}(\mathrm{a})=58 \mathrm{\mu eV}$. The European Physical Journal C, 78(10). arXiv:1806.00310 - Marsh, D.J.E., Fong, K.C., Lentz, E.W., Smejkal, L., Ali, M.N. (2019). Proposal to Detect Dark Matter using Axionic Topological Antiferromagnets. Physical Review Letters, 123(12), 121601. https://doi.org/10.1103/ PhysRevLett.123.121601

\section{Personal Response} How does the theory of axions differ from previous

WIMPs are comparatively heavy particles, with a mass around that of an atomic nucleus, and they are typically fermions: particles with spin. They are produced in the plasma and via interactions similar to the whe cosmic force. The WIMPs then cool, and we are left with slow moving, heavy beasts, which can smash into any nuclear material on Earth. Axions are very light particles, much lighter than even neutrinos, and they are bosons carrying no spin, which means they can get together in coherent, by processes of symmetry breaking which set the axion field into motion. Axion dark matter is the leftover oscillations from this process. What we aim to detect is not a particle smashing into something, but an oscillation disturbing something very slightly, but coherently. We do we tune like a radio until we find the right frequency. 